\title{
Gamma rays from cloud penetration at the base of AGN jets
}

\author{
A. T. Araudo ${ }^{1,2, \star}$, V. Bosch-Ramon ${ }^{3}$, and G. E. Romero ${ }^{1,2, \star \star}$ \\ 1 Instituto Argentino de Radioastronomía, C.C.5, (1894) Villa Elisa, Buenos Aires, Argentina \\ e-mail: aaraudo@fcaglp.unlp.edu.ar \\ 2 Facultad de Ciencias Astronómicas y Geofísicas, Universidad Nacional de La Plata, Paseo del Bosque, 1900 La Plata, Argentina \\ 3 Max Planck Institut für Kernphysik, Saupfercheckweg 1, 69117 Heidelberg, Germany
}

Received 31 March 2010 / Accepted 12 June 2010

\begin{abstract}
Context. Dense and cold clouds seem to populate the broad line region surrounding the central black hole in AGNs. These clouds may interact with the AGN jet base which could have observational consequences.

Aims. We study the gamma-ray emission produced by these jet-cloud interactions, and explore the conditions under which this radiation would be detectable.

Methods. We investigate the hydrodynamical properties of jet-cloud interactions and the resulting shocks, and develop a model to compute the spectral energy distribution of the emission generated by the particles accelerated in these shocks. We discuss our model in the context of radio-loud AGNs, with applications to two representative cases, the low-luminous Centaurus A and the powerful $3 \mathrm{C} 273$.

Results. Some fraction of the jet power may be channelled into gamma-ray energy, which is likely to be dominated by synchrotron self-Compton radiation, and have typical variability timescales similar to the cloud lifetime within the jet, which is longer than several hours. Many clouds can interact with the jet simultaneously leading to fluxes significantly higher than in one interaction, but then variability will be smoothed out.

Conclusions. Jet-cloud interactions may produce detectable gamma-rays in non-blazar AGNs that are transient in nearby lowluminous sources such as Cen A, and steady in the case of powerful objects of FR II type.
\end{abstract}

Key words. quasars: general - radiation mechanisms: non-thermal - gamma rays: general

\section{Introduction}

Active galactic nuclei (AGN) consist of an accreting supermassive black hole $(\mathrm{SMBH})$ in the center of a galaxy and sometimes exhibit powerful radio-emitting jets (Begelman et al. 1984). Radio-loud AGNs have continuum emission along the whole electromagnetic spectrum, from radio to gamma rays (e.g. Boettcher 2007). This radiation basically comes from the accretion disc and bipolar relativistic jets that originate close to the central SMBH. Radiation of accretion origin can be produced by the thermal plasma of either an optically-thick geometricallythin disc under efficient cooling (Shakura \& Sunyaev 1973), or an optically-thin geometrically-thick corona (e.g. Liang \& Thompson 1979). The emission from the jets is non-thermal and generated by a population of relativistic particles very likely accelerated in strong shocks, although other mechanisms are also possible (Rieger et al. 2007). This non-thermal emission is assumed to be produced by synchrotron and inverse Compton (IC) processes (e.g., Ghisellini et al. 1985), although hadronic models have been also considered to explain gamma-ray detections (e.g. Mannheim 1993; Mücke \& Protheroe 2001; Aharonian 2002).

In addition to continuum radiation, AGNs also exhibit optical and ultra-violet lines. Some of these lines are broad, emitted by gas moving with velocities $v_{\mathrm{g}}>1000 \mathrm{~km} \mathrm{~s}^{-1}$ and located in a small region close to the $\mathrm{SMBH}$, the so-called broad line region (BLR). The structure of this region is not well known, but

\footnotetext{
$\star$ Fellow of CONICET, Argentina.

$\star \star$ Member of CONICET, Argentina.
}

some models assume that the material in the BLR may have been formed by dense clouds confined within a hot $\left(T \sim 10^{8} \mathrm{~K}\right)$ external medium (Krolik et al. 1981) or by magnetic fields (Rees 1987). These clouds would be ionized by photons from the accretion disc producing the observed emission lines, which are broad because of the cloud motion within the SMBH potential well. An alternative model proposes that the broad lines are produced in the chromosphere of evolved stars (Penston 1988) present in the nuclear region of AGNs.

The presence of material surrounding the base of the jets in AGNs makes jet-medium interactions very likely. For instance, the interaction of BLR clouds with a jet in AGNs was already suggested by Blandford \& Königl (1979) as a mechanism for knot formation in the radio galaxy M87. Gamma-ray production by the interaction of a cloud from the BLR with a proton beam or a massive star with a jet were studied in the context of AGNs by Dar \& Laor (1997) and Bednarek \& Protheroe (1997), respectively.

In this work, we study the interaction of BLR clouds with the innermost jet in an AGN and its observable consequences at high energies. The approach adopted is similar to that followed in Araudo et al. (2009) for high-mass microquasars (for a general comparison between these sources and AGNs see Bosch-Ramon 2008), where the interaction of stellar wind clumps of the companion star with the microquasar jet was studied. In magnetic fields below equipartition with the jet kinetic energy (i.e. where the jet should be matter dominated), cloud penetration will lead to the formation of a relativistic bow shock in the jet and a slow shock inside the cloud. Electrons and protons can be efficiently 
accelerated in the bow shock and produce non-thermal emission, in situ by means of synchrotron and synchrotron self-Compton (SSC) mechanisms, and in the cloud through proton-proton $(p p)$ collisions. For magnetic fields well below equipartition, the SSC component becomes the dominant electron-cooling channel, leading to significant gamma-ray production. Since the bow shock downstream is almost at rest in the laboratory reference frame (RF), this emission will not be boosted significantly. The resulting spectrum and the achieved luminosities in one jet-cloud interaction depend strongly on the magnetic field, the location of the interaction region, the cloud size, and the jet luminosity. However, many clouds may be inside the jet simultaneously, and the BLR global properties, such as size and total number of clouds, would also be relevant. Depending on whether one cloud or many of them penetrate into the jet, the light curve will be flare-like or rather steady, respectively.

To explore the radiative outcomes of jet-cloud interactions in AGNs, we apply our model to both Faranoff-Riley galaxies I (FR I) and II (FR II). In particular, we consider Centaurus A (Cen A) and 3C 273, the nearest FR I and a close and very bright flat spectrum radio quasar (with FR II as parent population), respectively, as illustrative cases. Although in FR I the BLR is not well-detected, clouds with similar characteristics to those found in FR II galaxies may surround the SMBH (Wang et al. 1986; Risaliti 2010). Cen A has been detected at high- (HE) (Hartman et al. 1999; Abdo et al. 2010) and very high-energy (VHE) gamma rays (Aharonian et al. 2009), whereas 3C 273 has been detected so far only at HE gamma rays (Hartman et al. 1999; Abdo et al. 2010). We computed the contribution of jet-cloud interactions to the gamma-ray emission in these sources, and estimated the gamma-ray luminosity in a wide range of cases. We found that gamma rays from jet-cloud interactions should be detectable by present and future instrumentation in nearby lowluminous AGNs at $\mathrm{HE}$ and VHE, and in powerful and nearby quasars only at HE because the VHE radiation is absorbed by the dense nuclear photon fields. In the case of sources exhibiting boosted gamma rays (blazars), the isotropic radiation from jetcloud interactions will be masked by the jet beamed emission, which will not be the case in non-blazar sources.

The paper is organized as follows: in Sect. 2, the dynamics of jet-cloud interactions is described; in Sect. 3, a model for particle acceleration and emission is presented for one interaction, whereas in Sect. 4 the case of many clouds interacting with the jet is considered; in Sects. 5 and 6, the model is applied to FR I and FR II galaxies, focusing on the sources Cen A and 3C 273; finally, in Sect. 7, the results of this work are summarized and discussed. We adopt cgs units throughout this paper.

\section{The jet-cloud interaction}

For certain combinations of jet ram pressure, and cloud size and density, cloud-jet penetration is expected to occur. The details of the penetration process itself are complex. Here we do not treat them in detail, but just assume that penetration occurs if certain conditions are fulfilled. For low magnetic fields, a cloud inside the jet may represent a hydrodynamic situation in which a supersonic flow interacts with a body of approximately spherical shape at rest. The cloud, as long as it has not been accelerated by the jet ram pressure up to the jet speed $\left(v_{\mathrm{j}}\right)$, produces a perturbation in the jet medium in the form of a steady bow shock roughly at rest in the laboratory RF, moving at a velocity with respect to the jet RF approximately equal to $v_{\mathrm{j}}$. Since the cloud is not rigid, a wave propagates also through it. Since the cloud temperature is much lower, and the density much higher than in the

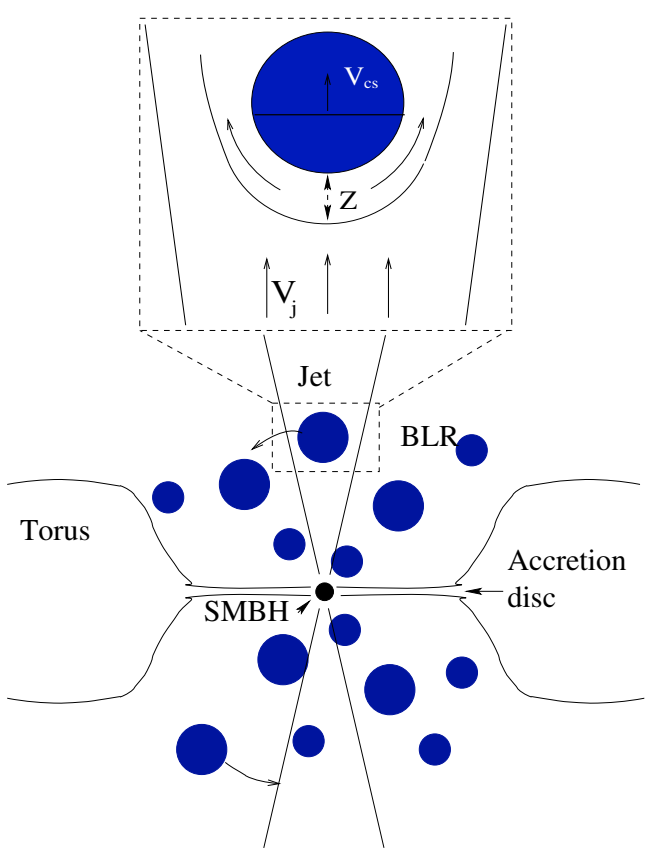

Fig. 1. Sketch, not to scale, of an AGN on the spatial scales of the BLR region. In the top part of the figure, the interaction between a cloud and the jet is also shown.

Table 1. Values assumed in this work for BLR clouds and jets.

\begin{tabular}{lc}
\hline \hline Description & Value \\
\hline Cloud size & $R_{\mathrm{c}}=10^{13} \mathrm{~cm}$ \\
Cloud density & $n_{\mathrm{c}}=10^{10} \mathrm{~cm}^{-3}$ \\
Cloud velocity & $v_{\mathrm{c}}=10^{9} \mathrm{~cm} \mathrm{~s}^{-1}$ \\
Cloud temperature & $T_{\mathrm{c}}=2 \times 10^{4} \mathrm{~K}$ \\
Jet Lorentz factor & $\Gamma=10$ \\
Jet half-opening angle & $\phi \approx 6^{\circ}$ \\
\hline
\end{tabular}

jet, this wave will be supersonic but much slower than the bow shock. The jet pressure exerts a force on the cloud leading to cloud acceleration along the axis, hydrodynamical instabilites, and, eventually, cloud fragmentation. In the following, the jetcloud interaction is described. Further discussion, and a proper account of the literature, can be found in Araudo et al. (2009). Sketchs of the jet-cloud interaction and the jet-BLR scenario are shown in Fig. 1.

We adopt here clouds with typical density $n_{\mathrm{c}}=10^{10} \mathrm{~cm}^{-3}$ and size $R_{\mathrm{c}}=10^{13} \mathrm{~cm}$ (Risaliti 2010). The velocity of the cloud is taken $v_{\mathrm{c}}=10^{9} \mathrm{~cm} \mathrm{~s}^{-1}$ (Peterson 2006). The jet Lorentz factor is fixed to $\Gamma=10$, implying $v_{\mathrm{j}} \approx c$, with a half-opening angle $\phi \approx 6^{\circ}$, i.e. the jet radius/height relation fixed to $R_{\mathrm{j}}=\tan (\phi) z=$ $0.1 z$. All these parameters are summarized in Table 1 and will not change in the paper; from them, the jet density $n_{\mathrm{j}}$ in the laboratoty RF can be estimated to be

$$
\begin{aligned}
n_{\mathrm{j}}=\frac{L_{\mathrm{j}}}{(\Gamma-1) m_{\mathrm{p}} c^{3} \sigma_{\mathrm{j}}} \approx & 8 \times 10^{4}\left(\frac{L_{\mathrm{j}}}{10^{44} \mathrm{erg} \mathrm{s}^{-1}}\right) \\
& \times\left(\frac{\Gamma-1}{9}\right)^{-1}\left(\frac{z}{10^{16} \mathrm{~cm}}\right)^{-2} \mathrm{~cm}^{-3}
\end{aligned}
$$

where $\sigma_{\mathrm{j}}=\pi R_{\mathrm{j}}^{2}$ and $L_{\mathrm{j}}$ is the kinetic power of the matterdominated jet. 
The jet ram pressure should not destroy the cloud before it has fully entered into the jet. This means that the time required by the cloud to penetrate into the jet should be

$t_{\mathrm{c}} \sim \frac{2 R_{\mathrm{c}}}{v_{\mathrm{c}}}=2 \times 10^{4}\left(\frac{R_{\mathrm{c}}}{10^{13} \mathrm{~cm}}\right)\left(\frac{v_{\mathrm{c}}}{10^{9} \mathrm{~cm} \mathrm{~s}^{-1}}\right)^{-1} \mathrm{~s}$,

which in turn should be shorter than the cloud lifetime inside the jet. To estimate this cloud lifetime, we first compute the time required by the shock in the cloud to cross it $\left(t_{\mathrm{cs}}\right)$. The velocity of this shock, $v_{\mathrm{cs}}$, can be derived by ensuring that the jet and the cloud shock ram pressures are equal, i.e., $(\Gamma-1) n_{\mathrm{j}} m_{\mathrm{p}} c^{2}=$ $n_{\mathrm{c}} m_{\mathrm{p}} v_{\mathrm{cs}}^{2}$, is valid as long as $v_{\mathrm{cs}} \ll c$. Then

$$
\begin{aligned}
v_{\mathrm{cs}} \sim \chi^{-1 / 2} c \sim & \times 10^{8}\left(\frac{n_{\mathrm{c}}}{10^{10} \mathrm{~cm}^{-3}}\right)^{-1 / 2} \\
& \times\left(\frac{z}{10^{16} \mathrm{~cm}}\right)^{-1}\left(\frac{L_{\mathrm{j}}}{10^{44} \mathrm{erg} \mathrm{s}^{-1}}\right)^{1 / 2} \mathrm{~cm} \mathrm{~s}^{-1},
\end{aligned}
$$

where $\chi$ is the cloud to jet density ratio, $n_{\mathrm{c}} / n_{\mathrm{j}}(\Gamma-1)$. This yields a cloud shocking time of

$$
\begin{aligned}
t_{\mathrm{cs}} \sim \frac{2 R_{\mathrm{c}}}{v_{\mathrm{cs}}} \simeq & 7 \times 10^{4}\left(\frac{R_{\mathrm{c}}}{10^{13} \mathrm{~cm}}\right)\left(\frac{n_{\mathrm{c}}}{10^{10} \mathrm{~cm}^{-3}}\right)^{1 / 2} \\
& \times\left(\frac{z}{10^{16} \mathrm{~cm}}\right)\left(\frac{L_{\mathrm{j}}}{10^{44} \mathrm{erg} \mathrm{s}^{-1}}\right)^{-1 / 2} \mathrm{~s} .
\end{aligned}
$$

Therefore, for a penetration time $\left(t_{\mathrm{c}}\right)$ at least as short as $\sim t_{\mathrm{cs}}$, the cloud will remain an effective obstacle for the jet flow. Setting $t_{\mathrm{c}} \sim t_{\mathrm{cs}}$, we obtain a minimum value for $\chi$ and hence for $z$.

Hydrodynamical instabilities produced by the interaction with the jet material affect the cloud. First of all, the jet exerts a force on the cloud through the contact discontinuity. The acceleration applied to the cloud can be estimated from the jet ram pressure $P_{\mathrm{j}}$, and the cloud section $\sigma_{\mathrm{c}} \sim \pi R_{\mathrm{c}}^{2}$ and mass $M_{\mathrm{c}} \sim(4 / 3) \pi R_{\mathrm{c}}^{3} n_{\mathrm{j}} m_{\mathrm{p}}$

$g=\frac{P_{\mathrm{j}} \sigma_{\mathrm{c}}}{M_{\mathrm{c}}} \sim \frac{3}{4} \frac{c^{2}}{\chi R_{\mathrm{c}}}=\frac{3}{2} \frac{v_{\mathrm{cs}}}{t_{\mathrm{cs}}}$.

Given the acceleration exerted by the jet in the cloud, RayleighTaylor (RT) instabilities will develop in the cloud, at the jet contact discontinuity, on a timescale

$t_{\mathrm{RT}} \sim \sqrt{\frac{l}{g}}=\sqrt{\frac{4 \chi l R_{\mathrm{c}}}{3 c^{2}}}$,

where the instability length $l$ is the spatial scale of the perturbation. For perturbations in the size of the cloud $l \sim R_{\mathrm{c}}$ associated with cloud significant disruption, one finds that $t_{\mathrm{RT}} \sim t_{\mathrm{cs}}$.

In addition to RT instabilities, Kelvin-Helmholtz (KH) instabilities also grow within the cloud walls in contact with the shocked jet material surrounding the cloud. Accounting for the high relative velocity, $v_{\text {rel }} \lesssim v_{\mathrm{j}}$, one obtains

$t_{\mathrm{KH}} \gtrsim \sqrt{\frac{l}{g_{\mathrm{rel}}}}=\frac{\chi l}{c}$,

where $g_{\text {rel }} \sim c^{2} / \chi l$. For $l \sim R_{\mathrm{c}}$, we obtain $t_{\mathrm{KH}} \gtrsim t_{\mathrm{cs}}$. In the previous estimates of $t_{\mathrm{RT}}$ and $t_{\mathrm{KH}}$, we did not take into account the effect of the magnetic field (e.g. Blake 1972), since we assume that it is dynamically negligible. We note that, given $g$, the time to accelerate the cloud to the shock velocity $v_{\mathrm{cs}}$ is $\sim t_{\mathrm{cs}}$. However,

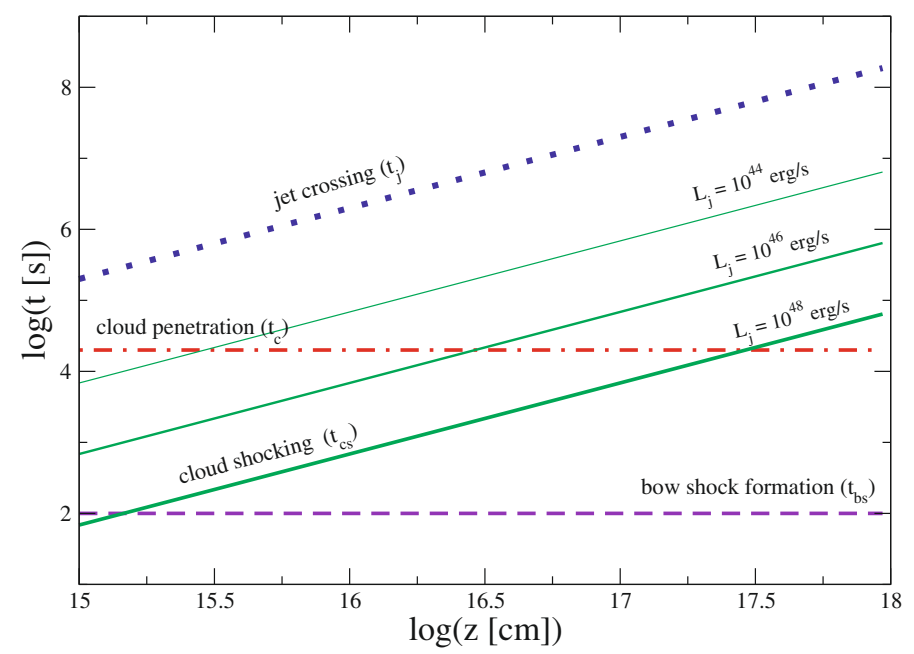

Fig. 2. The jet crossing (blue dotted line), cloud penetration (red dotdashed line), bow-shock formation (violet dashed line), and cloud shocking (green solid lines) times are plotted; all of them have been calculated using the values given in Table 1 . The time $t_{\mathrm{cs}}$ is plotted for $L_{\mathrm{j}}=10^{44}, 10^{46}$, and $10^{48} \mathrm{erg} \mathrm{s}^{-1}$.

the timescale to accelerate the cloud to $v_{\mathrm{j}}$ is $\gg t_{\mathrm{cs}}$ provided that $v_{\mathrm{j}} \gg v_{\mathrm{cs}}$, and before this occurs, the cloud will likely fragment.

Finally, two additional timescales are relevant to our study, the bow-shock formation time, $t_{\mathrm{bs}}$, and the time required by the cloud to cross the jet, $t_{\mathrm{j}}$. The timescale $t_{\mathrm{bs}}$ can be roughly estimated assuming that the shock downstream has a cylindrical shape with one of the bases being the bow shock, relativistic shock jump conditions, equal particle injection and escape rates, and a escape velocity similar to the sound speed $\sim c / \sqrt{3}$ (for a relativistic plasma). This yields a shock-cloud separation distance of $Z \sim 0.3 R_{\mathrm{c}}$, which implies that

$t_{\mathrm{bs}} \sim \frac{Z}{c}=10^{2}\left(\frac{R_{\mathrm{c}}}{10^{13} \mathrm{~cm}}\right) \mathrm{s}$.

Since in general $t_{\mathrm{bs}} \ll t_{\mathrm{cs}}$, we can assume that the bow shock is in the steady regime. The jet crossing time $t_{\mathrm{j}}$ can be characterized by

$t_{\mathrm{j}} \sim \frac{2 R_{\mathrm{j}}}{v_{\mathrm{c}}}=2 \times 10^{6}\left(\frac{z}{10^{16} \mathrm{~cm}}\right)\left(\frac{v_{\mathrm{c}}}{10^{9} \mathrm{~cm} \mathrm{~s}^{-1}}\right)^{-1} \mathrm{~s}$.

We note that if the cloud lifetime is $<t_{j}$, the number of clouds inside the jet will be smaller than expected from the BLR properties alone.

To summarize the dynamics of the jet-cloud interaction, we plot in Fig. 2 the $t_{\mathrm{cs}}$ (for different $L_{\mathrm{j}}$ ), $t_{\mathrm{j}}, t_{\mathrm{c}}$, and $t_{\mathrm{bs}}$ as a function of $z$. As shown in the figure, for some values of $z$ and $L_{j}$ the cloud may be destroyed by the jet before full penetration, i.e. $t_{\mathrm{cs}}<t_{\mathrm{c}}$. This is a constraint to determine the height $z$ of the jet at which the cloud can penetrate into it. We also note that, in general, $t_{\mathrm{bs}}$ is much shorter than any other timescale.

\subsection{The interaction height}

The cloud can fully penetrate into the jet if the cloud lifetime after jet impact is longer than the penetration time (the weaker condition that jet lateral pressure is $<n_{\mathrm{c}} m_{\mathrm{p}} v_{\mathrm{c}}^{2}$ is then automatically satisfied). This determines the minimum interaction height, $z_{\text {int }}$, to avoid cloud disruption before full penetration. This interaction also, cannot occur within the jet formation region, 
$z_{0} \sim 100 R_{\mathrm{g}} \approx 1.5 \times 10^{15}\left(M_{\mathrm{bh}} / 10^{8} M_{\odot}\right) \mathrm{cm}$ (Junor et al. 1999). For BLR-jet interaction and cloud penetration to occur, the size of the BLR should be $R_{\mathrm{blr}}>z_{0}$ and $z_{\text {int }}$.

The lifetime of the cloud depends on the fragmentation time, which is strongly linked to, but longer than, $t_{\mathrm{cs}}$. The value of $z_{\text {int }}$ can then be estimated by setting $t_{\mathrm{c}} \lesssim t_{\mathrm{cs}}$, since the cloud should enter the jet before being significantly distorted by the impact of the latter. Once shocked, the cloud may suffer lateral expansion and conduction heating, which can speed up fragmentation due to instabilities. In this work, we choose $z_{\text {int }}$ fixing $t_{\mathrm{cs}}=2 t_{\mathrm{c}}$

$$
\begin{aligned}
z_{\text {int }} \approx & 5 \times 10^{15}\left(\frac{v_{\mathrm{c}}}{10^{9} \mathrm{~cm} \mathrm{~s}^{-1}}\right)^{-1}\left(\frac{n_{\mathrm{c}}}{10^{10} \mathrm{~cm}^{-3}}\right)^{-1 / 2} \\
& \times\left(\frac{L_{\mathrm{j}}}{10^{44} \mathrm{erg} \mathrm{s}^{-1}}\right)^{1 / 2} \mathrm{~cm} .
\end{aligned}
$$

We note that the available power in the bow shock is $L_{\mathrm{bs}} \sim$ $\left(\sigma_{\mathrm{c}} / \sigma_{\mathrm{j}}\right) L_{\mathrm{j}} \propto z^{-2}$. Therefore, the most luminous individual jetcloud interaction will take place at $z \sim z_{\text {int }}$.

The BLR size can be estimated using an empirical relation derived for sources with a well-established BLR, i.e. FR II radio galaxies. This relation is in general of type $R_{\mathrm{blr}} \propto L_{\mathrm{blr}}^{\alpha}$, where $L_{\text {blr }}$ is the luminosity of the BLR and $\alpha \sim 0.5-0.7$ (e.g. Kaspi et al. 2005, 2007; Peterson et al. 2005; Bentz et al. 2006). In this paper, we use the relations

$R_{\mathrm{blr}} \sim 6 \times 10^{16}\left(\frac{L_{\mathrm{blr}}}{10^{44} \mathrm{erg} \mathrm{s}^{-1}}\right)^{0.7} \mathrm{~cm}$,

and

$R_{\mathrm{blr}} \sim 2.5 \times 10^{16}\left(\frac{L_{\mathrm{blr}}}{10^{44} \mathrm{erg} \mathrm{s}^{-1}}\right)^{0.55} \mathrm{~cm}$,

from Kaspi et al. (2005, 2007).

In Fig. 3, we show the relation of $z_{\text {int }}$ and $R_{\mathrm{blr}}$ with $L_{\mathrm{j}}$, assuming that $L_{\mathrm{blr}}$ is $10 \%$ of the disc luminosity, which is assumed here to equal $L_{\mathrm{j}}$. As seen in the figure, for reasonable parameters, the condition $z_{\text {int }}<R_{\mathrm{blr}}$ is fulfilled for a wide range of $L_{\mathrm{j}}$. Figure 3 also shows the relation between $z_{0}$ and $M_{\mathrm{bh}}$, which shows that for $M_{\mathrm{bh}} \gtrsim 10^{9} M_{\odot}$ the jet may not even be (fully) formed at the BLR scales at the lowest $L_{\mathrm{j}}$-values.

\section{Non-thermal particles and their emission}

In the bow and cloud shocks, particles can be accelerated by means of diffusive shock acceleration (Bell 1978). However, the bow shock should be more efficient in accelerating particles than the shock in the cloud because $v_{\mathrm{bs}} \gg v_{\mathrm{cs}}$. In addition, the cloud shock luminosity is lower than the bow-shock luminosity by $\sim 1 /\left(2 \chi^{1 / 2}\right)$. For these reasons, we focus here on the particle acceleration in the bow shock. In this section, we briefly describe the injection and evolution of particles, and their emission, highlighting aspects that are specific to AGNs. The details of the emitting processes considered here (synchrotron, IC, and $p p$ interactions) can be found in Araudo et al. (2009) and references therein.

First, one can estimate the non-thermal luminosity, $L_{\mathrm{nt}}$, injected at $z_{\text {int }}$ in the bow shock in the form of relativistic electrons or protons

$$
\begin{aligned}
L_{\mathrm{nt}}=\eta_{\mathrm{nt}} L_{\mathrm{bs}} \approx & 4 \times 10^{39}\left(\frac{\eta_{\mathrm{nt}}}{0.1}\right)\left(\frac{R_{\mathrm{c}}}{10^{13} \mathrm{~cm}}\right)^{2} \\
& \times\left(\frac{L_{\mathrm{j}}}{10^{44} \mathrm{erg} \mathrm{s}^{-1}}\right) \mathrm{erg} \mathrm{s}^{-1} .
\end{aligned}
$$

Page 4 of 8

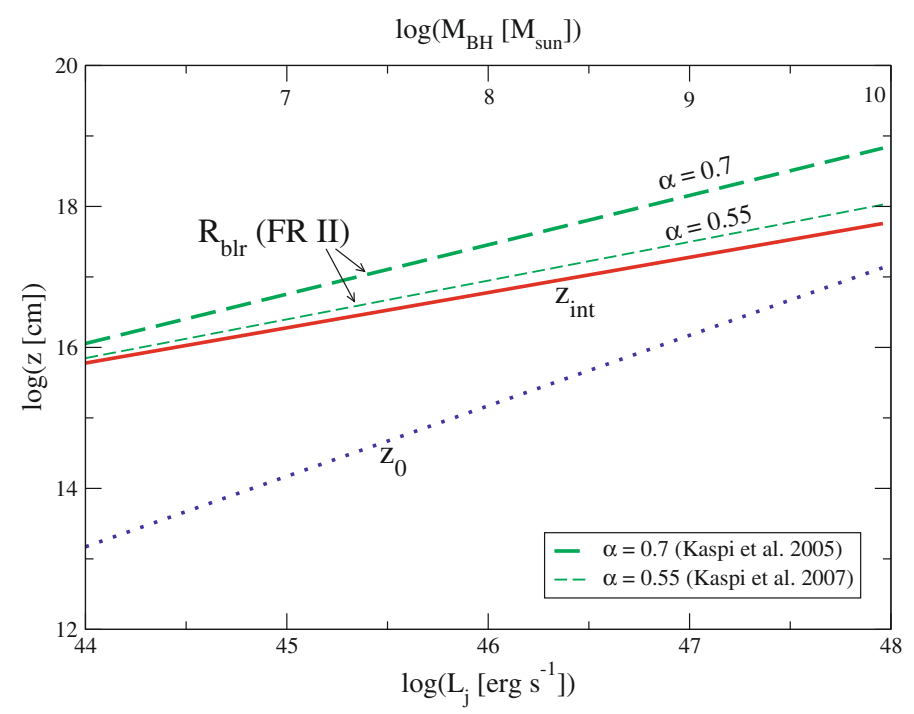

Fig. 3. The interaction height, $z_{\text {int }}$ (red solid line), and the size of the BLR, $R_{\mathrm{blr}}$ (green dashed line), for different values of $L_{\mathrm{j}}$ (bottom horizontal axis). We have derived $R_{\mathrm{blr}}\left(L_{\mathrm{j}}\right)$ fixing $L_{\mathrm{blr}}=0.1 L_{\mathrm{j}}$ and plotted $R_{\mathrm{blr}}$ using Eqs. (11) and (12). In the same plot, the height of the jet base, $z_{0}$ (blue dotted line), is plotted as a function of $M_{\mathrm{BH}}$ (top horizontal axis).

The accelerator/emitter magnetic field in the bow-shock RF $(B)$ can then, be determined by relating $U_{\mathrm{B}}=\eta_{\mathrm{B}} U_{\mathrm{nt}}$, where $U_{\mathrm{B}}=$ $B^{2} / 8 \pi$ and $U_{\mathrm{nt}}=L_{\mathrm{nt}} /\left(\sigma_{\mathrm{c}} c\right)$ are the magnetic and the non-thermal energy densities, respectively. For leptonic emission and to avoid supression of the IC channel, high gamma-ray outputs require $\eta_{\mathrm{B}}$ well below 1 . In this context, $B$ can be parametrized to be

$B \approx 10\left(\frac{\eta_{\mathrm{B}}}{0.01}\right)\left(\frac{v_{\mathrm{c}}}{10^{9} \mathrm{~cm} \mathrm{~s}^{-1}}\right)^{2}\left(\frac{n_{\mathrm{c}}}{10^{10} \mathrm{~cm}^{-3}}\right) \mathrm{G}$.

Regarding the acceleration mechanism, since the bow shock is relativistic and the treatment of these shocks is complex (see Achterberg et al. 2001), we adopt a prescription for the acceleration rate of

$\dot{E}_{\mathrm{acc}}=0.1 q B c$

similar to that in the relativistic termination shock of the Crab pulsar wind (de Jager et al. 1996).

Particles are affected by different losses that balance the energy gain from acceleration. The electron loss mechanisms include escape downstream, relativistic bremsstrahlung, synchrotron emission, and external Compton (EC) and SSC. We note that $B, L_{\mathrm{nt}}$, and the accelerator/emitter size at $z_{\text {int }}$ are constant for different $L_{\mathrm{j}}$ and fixed $\eta_{\mathrm{B}}$ and $\eta_{\mathrm{nt}}$, and only $L_{\mathrm{blr}}$ and $L_{\mathrm{d}}$ are expected to change with $L_{\mathrm{j}}$. Therefore, as long as the external photon fields are negligible, the maximum electron energy at $z_{\text {int }}$ does not change for different jet powers.

In Fig. 4, the leptonic cooling timescales are plotted with the escape time and the acceleration time for a bow shock located at $z_{\text {int }}$. A value of $\eta_{\mathrm{B}}$ equal to 0.01 has been adopted. The SSC cooling timescale is plotted for the steady state. The escape time downstream from the relativistic bow shock is taken to be

$t_{\mathrm{esc}} \sim \frac{3 R_{\mathrm{c}}}{c}=10^{3}\left(\frac{R_{\mathrm{c}}}{10^{13} \mathrm{~cm}}\right) \mathrm{s}$.

Synchrotron and EC/SSC are the dominant processes in the high-energy part of the electron population, relativistic bremsstrahlung is negligible for any energy, and electron escape is relevant in the low-energy part. This yields a break in 


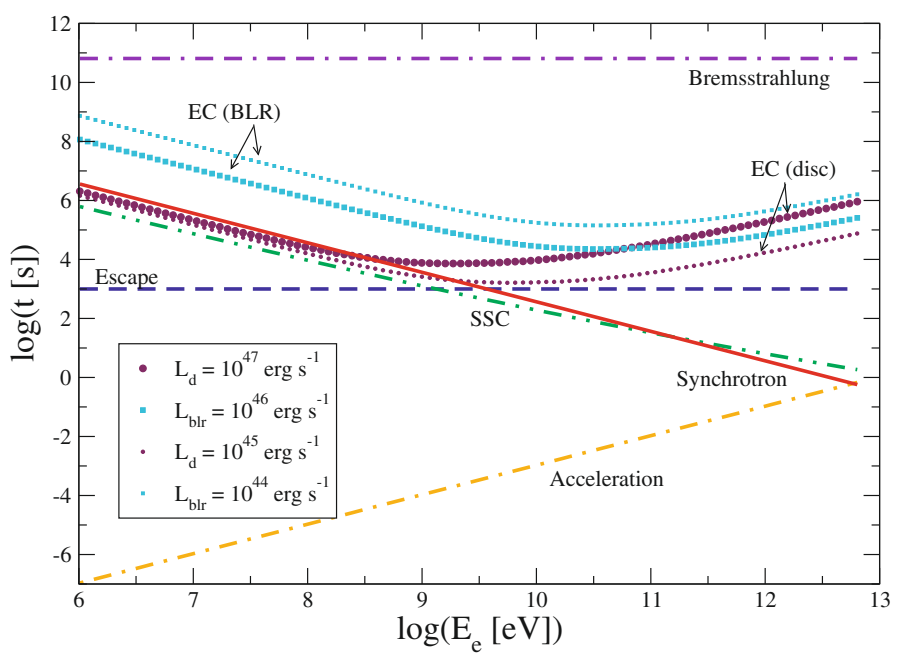

Fig. 4. Acceleration gain (orange dot-dashed line), escape (blue dashed line), and cooling lepton timescales are plotted. SSC (green two-dotdashed line) is plotted when the steady state is reached and EC for the both BLR (turquoise square line) and disc (maroon dotted line) photon fields are shown for the conditions of faint (BLR: $10^{44}$; disc: $10^{45} \mathrm{erg} \mathrm{s}^{-1}$ ) and bright sources (BLR: $10^{46}$; disc: $10^{47} \mathrm{erg} \mathrm{s}^{-1}$ ). Synchrotron (red solid line) and relativistic bremsstrahlung (violet dottwo-dashed line) are also plotted. We have fixed $\eta_{\mathrm{B}}=0.01$. For protons, the $p p$ cooling timescale is not shown for clarity, although it is similar to that of relativistic bremsstrahlung.

the electron energy distribution at the energy for which the synchrotron/IC time and the escape time are equal. The Thomson to $\mathrm{KN}$ transition is clearly seen in the EC cooling curves, but is much smoother in the SSC case. The maximun electrons energy are around several $\mathrm{TeV}$. Given the similar cooling timescale for electrons by means of relativistic bremsstrahlung and protons by $p p$ collisions $\left(t_{\text {brems } / p p} \sim 10^{15} / n \mathrm{~s}\right.$, where $n$ is the target density), protons will not cool efficiently in the bow shock. Photomeson production can also be discarded as a relevant proton cooling mechanism in the bow shock due to the relatively low achievable proton energies and photon densities. The maximum energy of protons is constrained by equating the acceleration time and the time needed to diffuse out of the bow shock. Assuming Bohm diffusion, $t_{\mathrm{diff}}=3 Z^{2} / 2 r_{\mathrm{g}} c$ (where $r_{\mathrm{g}}$ is the particle gyroradius), the maximum proton energy is

$E_{\mathrm{p}}^{\max } \sim 0.1 q B R_{\mathrm{c}}=5 \times 10^{3}\left(\frac{B}{10 \mathrm{G}}\right)\left(\frac{R_{\mathrm{c}}}{10^{13} \mathrm{~cm}}\right) \mathrm{TeV}$.

Electrons and protons are injected into the bow shock region following a power law in energy of index 2.2 (Achterberg et al. 2001) with an exponential cutoff at the maximum electron/proton energy. The injection luminosity is $L_{\mathrm{nt}}$.

To first order, the electron evolution can be computed assuming homogeneous conditions, following therefore a one-zone approximation with all the mentioned cooling and escape processes. The formulae for all the relevant radiative mechanisms, as well as the solved electron evolution differential equation, can be found in Araudo et al. (2009). In some cases, SSC is the dominant cooling channel at high energies. In that case, the calculations have to be performed numerically by separating the evolution of the electron population into different time steps. In each step, the radiation field is updated by accounting for the synchrotron emission produced in the previous step until the steady state is reached. The duration of each step should be shorter for the earlier phases of the evolutionary state to account properly for the rise in the synchrotron energy density of the emitter.
Once the steady-state electron distribution in the bow shock is computed, the spectral energy distribution (SED) of the non-thermal radiation can be calculated. The synchrotron selfabsorption effect has to be taken into account, which will affect the low energy band of the synchrotron emission. At gamma-ray energies, photon-photon absorption due to the disc and the BLR radiation should be considered, the internal absorption due to synchrotron radiation being negligible. Given the typical BLR and disc photon energies, $\sim 10 \mathrm{eV}$ and $\sim 1 \mathrm{keV}$, respectively, gamma rays beyond $1 \mathrm{GeV}$ and $100 \mathrm{GeV}$ can be strongly affected by photon-photon absorption. On the other hand, in most cases photons of energies between $100 \mathrm{MeV}$ and $1 \mathrm{GeV}$ will escape the dense disc photon field.

Although proton cooling is negligible in the bow-shock region, it may be significant in the cloud. Protons can penetrate into the cloud if $t_{\mathrm{esc}}>t_{\text {diff }}$, which yields a minimum energy to reach the cloud of $E_{\mathrm{p}} \sim 0.4 E_{\mathrm{p}}^{\max }$. These protons will radiate in the form of gamma rays only a fraction $\sim 3 \times$ $10^{-4}\left(R_{\mathrm{c}} / 10^{13} \mathrm{~cm}\right)\left(t_{\mathrm{pp}} / 10^{5} \mathrm{~s}\right)^{-1}$ of their energy, implying that the process is rather inefficient. The reason is that these protons cannot be efficiently confined and cross the cloud at a velocity $\sim c$. For further details of the proton energy distribution in the cloud, we refer to Araudo et al. (2009).

\section{Many clouds inside the jet}

Clouds fill the BLR, and many of them may be simultaneously inside the jet at different $z$, each of them producing non-thermal radiation. Therefore, the total luminosity may be much larger than that produced by just one interaction, which is $\sim L_{\mathrm{nt}}$. The number of clouds within the jets, at $z \leq R_{\mathrm{blr}}$, can be computed from the jet $\left(V_{\mathrm{j}}\right)$ and cloud $\left(V_{\mathrm{c}}\right)$ volumes, resulting in

$N_{\mathrm{c}}^{\mathrm{j}}=2 f \frac{V_{\mathrm{j}}}{V_{\mathrm{c}}} \sim 9\left(\frac{L_{\mathrm{j}}}{10^{44} \mathrm{erg} \mathrm{s}^{-1}}\right)^{2}\left(\frac{R_{\mathrm{c}}}{10^{13} \mathrm{~cm}}\right)^{-3}$,

where the factor 2 accounts for the two jets and $f \sim 10^{-6}$ is the filling factor of clouds in the whole BLR (Dietrich et al. 1999). In reality, $N_{\mathrm{c}}^{\mathrm{j}}$ is correct if one neglects that the cloud disrupts and fragments, and eventually dilutes inside the jet. For instance, Klein et al. (1994) estimated a shocked cloud lifetime of several $t_{\mathrm{cs}}$, and Shin et al. (2008) found that even a weak magnetic field in the cloud can significantly increase its lifetime. Finally, even under cloud fragmentation, strong bow shocks can form around the cloud fragments before these have accelerated close to $v_{\mathrm{j}}$. All this makes the real number of interacting clouds inside the jet difficult to estimate, but it should be between $\left(t_{\mathrm{cs}} / t_{\mathrm{j}}\right) N_{\mathrm{c}}^{\mathrm{j}}$ and $N_{\mathrm{c}}^{\mathrm{j}}$.

The presence of many clouds inside the jet, not only at $z_{\text {int }}$ but also at higher $z$, implies that the total non-thermal luminosity available in the BLR-jet intersection region is

$$
\begin{aligned}
L_{\mathrm{nt}}^{\mathrm{tot}} & \sim 2 \int^{R_{\mathrm{blr}}} \frac{\mathrm{d} N_{\mathrm{c}}^{\mathrm{j}}}{\mathrm{d} z} L_{\mathrm{nt}}(z) \mathrm{d} z \\
& \sim 2 \times 10^{40}\left(\frac{\eta_{\mathrm{nt}}}{0.1}\right)\left(\frac{R_{\mathrm{c}}}{10^{13} \mathrm{~cm}}\right)^{-1}\left(\frac{L_{\mathrm{j}}}{10^{44} \mathrm{erg} \mathrm{s}^{-1}}\right)^{1.7}
\end{aligned}
$$

where $\mathrm{d} N_{\mathrm{c}}^{\mathrm{j}}$ is the number of clouds located in a jet volume $\mathrm{d} V_{\mathrm{j}}=$ $\pi(0.1 z)^{2} \mathrm{~d} z$. In both Eqs. (18) and (19), $L_{\mathrm{blr}}$ has been fixed to $0.1 L_{\mathrm{j}}$, as approximately found in FR II galaxies, and $R_{\mathrm{blr}}$ has been derived using Eq. (11).

In Fig. 5, we show estimates of the gamma-ray luminosity when many clouds interact simultanously with the jet. For this, we have followed a simple approach assuming that most of the 


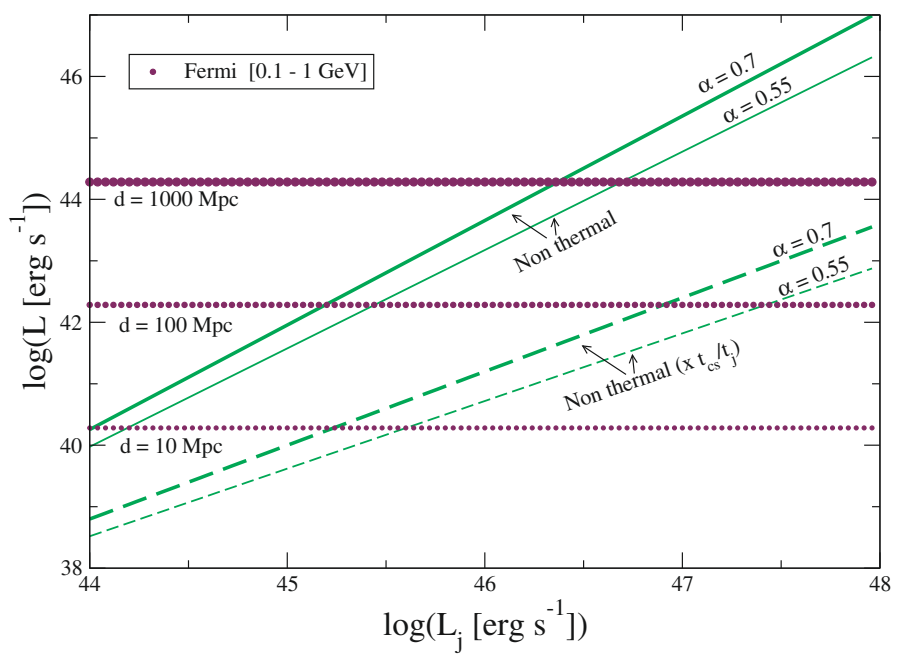

Fig. 5. Upper limits to the gamma-ray luminosity produced by $N_{\mathrm{c}}^{\mathrm{j}}$ clouds inside the jet as a function of $L_{\mathrm{j}}$ in FR II sources. Two cases are plotted, one assuming that clouds cross the jet without disruption (green solid lines), and one in which the clouds are destroyed in a time as short as $t_{\mathrm{cs}}$ (green dashed lines). The thick (solid and dashed) and thin (solid and dashed) lines correspond to $R_{\mathrm{blr}} \propto L_{\mathrm{blr}}^{0.7}$ (Kaspi et al. 2007) and $R_{\mathrm{blr}} \propto L_{\mathrm{blr}}^{0.55}$ (Kaspi et al. 2005), respectively. In addition, the sensitivity levels of Fermi in the range $0.1-1 \mathrm{GeV}$ (maroon dotted lines) are plotted for three different distances $d=10,100$, and $1000 \mathrm{Mpc}$.

non-thermal luminosity is converted into gamma rays. This will be the case as long as the escape and synchrotron cooling time are longer than the IC cooling time $(\mathrm{EC}+\mathrm{SSC})$ at the highest electron energies. Given the little information about the BLR available for FR I sources, we do not specifically consider these sources here.

In the next two sections, we present more detailed calculations by applying the model presented in Sect. 3 to two characteristic sources, Cen A (FR I, one interaction) and 3C 273 (FR II, many interactions).

\section{Application to FR I galaxies: Cen A}

At a distance $d \approx 3.7 \mathrm{Mpc}$, Cen $\mathrm{A}$ is the closest AGN (Israel 1998). It has been classified as both an FR I radio galaxy and a Seyfert 2 optical object. The mass of the black hole is $\approx 6 \times 10^{7} M_{\odot}$ (Marconi et al. 2000). The angle between the jets and the line of sight is large $>50^{\circ}$ (Tingay et al. 1998), thus the jet radiation towards the observer should not experience strong beaming. The jets of Cen A are disrupted on kpc scales, forming two giant radio lobes that extend $\sim 10^{\circ}$ across the southern sky. At optical wavelenghts, the nuclear region of Cen A is obscured by a dense region of gas and dust, probably as a consequence of a recent merger (Thomson 1992; Mirabel et al. 1999). At higher energies, Chandra and XMM-Newton detected continuum X-ray emission originating in from the nuclear region, with a luminosity $\sim 5 \times 10^{41} \mathrm{erg} \mathrm{s}^{-1}$ at energies of 2-7 keV (Evans et al. 2004). These X-rays could be produced by the accretion flow and the inner jet, although their origin remains unclear. In the $\mathrm{GeV}$ range, Cen A was detected above $200 \mathrm{MeV}$ by Fermi, with a bolometric luminosity of $\approx 4 \times 10^{40} \mathrm{erg} \mathrm{s}^{-1}$ (Abdo et al. 2009), and above $\sim 200 \mathrm{GeV}$ by HESS, with a bolometric luminosity of $\approx 3 \times 10^{39} \mathrm{erg} \mathrm{s}^{-1}$ (Aharonian et al. 2009). In both cases, this $\mathrm{HE}$ emission is associated with the nuclear region. Cen $\mathrm{A}$ has been proposed to be a source of ultra HE cosmic rays (Romero et al. 1996).

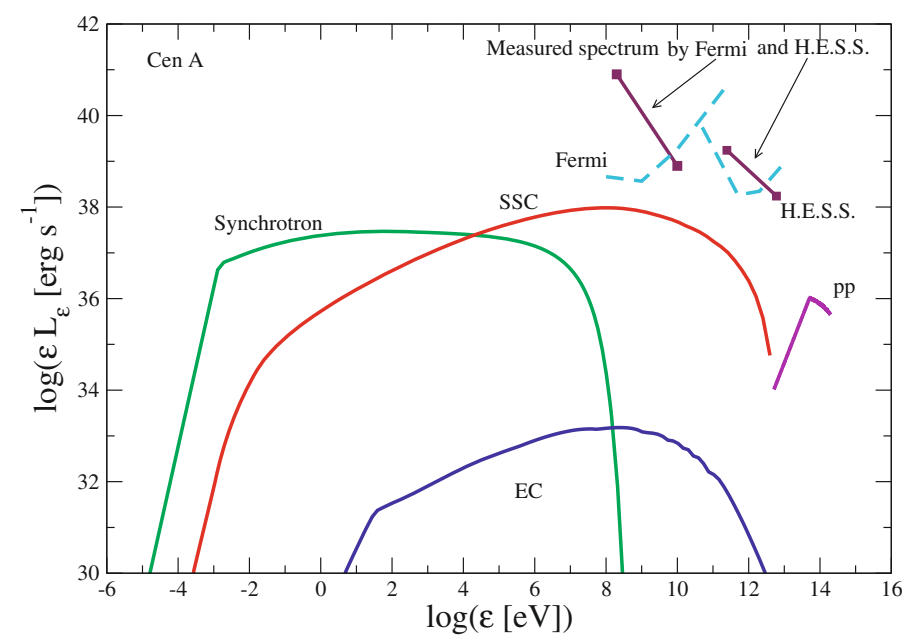

Fig. 6. Computed SED for one jet-cloud interaction at $z_{\text {int }}$ in Cen A. We also show the SEDs of the detected emission by Fermi and HESS, as well as the sensitivity curves of these instruments.

A BLR has not yet been detected in Cen A (Alexander et al. 1999), although this may be a consequence of the optical obscuration produced by the dust lane. One can still assume that clouds surround the SMBH in the nuclear region (Wang et al. 1986; Risaliti et al. 2002) but, because of the low luminosities of the accretion disc, it is not expected that the photoionization of these clouds will be efficient enough to produce lines. Since no emission from these clouds is assumed, we only consider the EC scattering with photons from the accretion flow.

We adopt here a jet power for Cen A of $L_{\mathrm{j}}=10^{44} \mathrm{erg} \mathrm{s}^{-1}$. From this value, and the values given in Sect. 2 for the remaining parameters of the jet and the cloud, $z_{\text {int }}$ results in $\approx 5 \times 10^{15} \mathrm{~cm}$. At this jet height, the emission produced by the interaction between one cloud and the jet is calculated assuming a $\eta_{\mathrm{B}}=0.01$, and the corresponding SED is presented in Fig. 6. As mentioned in Sect. 3, the low-energy band of the synchrotron spectrum is self-absorbed at energies below $\sim 10^{-4} \mathrm{eV}$. At gamma-ray energies, photon-photon absorption is negligible due to the weak ambient photon fields (e.g. Rieger \& Aharonian 2009; Araudo et al. 2009, 2010a,b). At high energies, SSC dominates the radiative output, with the computed luminosity above $100 \mathrm{MeV}$ being $\sim 2 \times 10^{39} \mathrm{erg} \mathrm{s}^{-1}$, and above $100 \mathrm{GeV}$ about 10 times less. These luminosities are below the sensitivity of Fermi and HESS and one order of magnitude lower than the observed ones. We note however that $L_{\mathrm{nt}} \propto R_{\mathrm{c}}^{2}$, and for slightly larger clouds, $L_{\mathrm{nt}}$ may increase to detectable levels. The penetration of a large clump into the base of the jet of Cen A would lead to a flare with a duration of about one day.

\section{Application to FR II galaxies: $3 C 273$ (off-axis)}

At a distance of $d=6.7 \times 10^{2} \mathrm{Mpc}, 3 \mathrm{C} 273$ is a powerful radio-loud AGN (Courvoisier 1998) with a SMBH mass $M_{\mathrm{BH}} \sim 7 \times 10^{9} M_{\odot}$ (Paltani \& Türler 2005). The angle of the jet to the line of sight is small, $\approx 6^{\circ}$, which implies that $3 \mathrm{C} 273$ is a blazar (Jolley et al. 2009). The whole spectrum of this source exhibits variability (e.g. Pian et al. 1999) from years (radio) to few hours (gamma rays). At high energies, 3C 273 was the first blazar AGN detected in the MeV band by the COS-B satellite and, later on, by EGRET (Hartman et al. 1999). This source was also detected at GeV energies by Fermi and AGILE, but has not yet been detected in the $\mathrm{TeV}$ range. Given the jet luminosity 


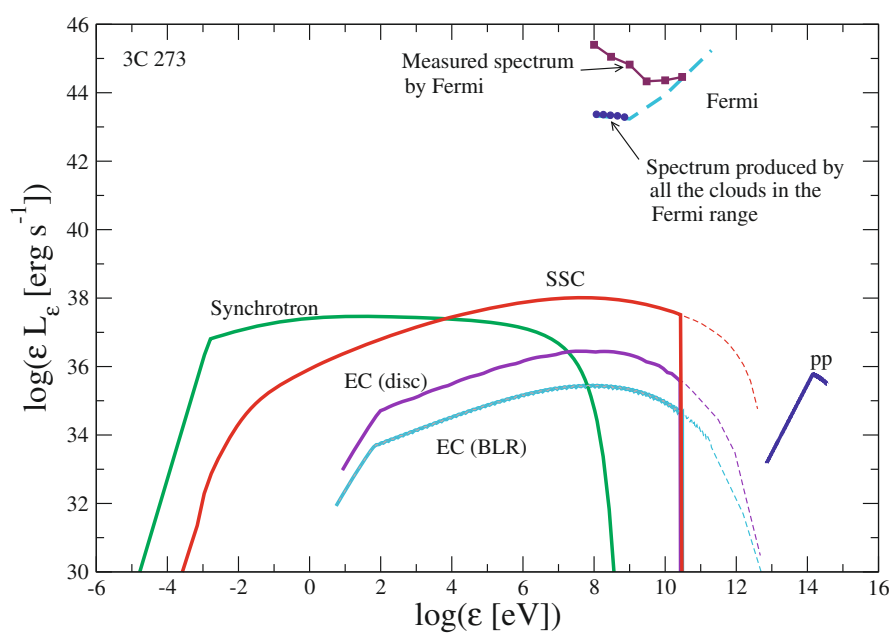

Fig. 7. Computed SED for one jet-cloud interaction at $z_{\text {int }}$ in 3C 273. The emission in the $0.1-1 \mathrm{GeV}$ range from many clouds inside the jet is also shown, together with the sensitivity level of Fermi and the observed SED above $200 \mathrm{MeV}$.

Table 2. Adopted parameters for Cen A and 3C 273.

\begin{tabular}{|c|c|c|}
\hline & Cen A & $3 \mathrm{C} 273$ \\
\hline Distance $[\mathrm{Mpc}]$ & 3.7 & $6.7 \times 10^{2}$ \\
\hline Black hole mass $\left[M_{\odot}\right]$ & $6 \times 10^{7}$ & $7 \times 10^{9}$ \\
\hline Inclination angle $\left[{ }^{\circ}\right]$ & $>50$ & $\sim 15$ \\
\hline Jet luminosity [erg s${ }^{-1}$ ] & $10^{44}$ & $4 \times 10^{47}$ \\
\hline Disc luminosity [erg s${ }^{-1}$ ] & $5 \times 10^{41}$ & $2 \times 10^{46}$ \\
\hline Disc photon energy ${ }^{\star}[\mathrm{eV}]$ & $\sim 5 \times 10^{3}$ & 54 \\
\hline BLR luminosity $L_{\mathrm{blr}}\left[\mathrm{erg} \mathrm{s}^{-1}\right]$ & - & $4 \times 10^{45}$ \\
\hline
\end{tabular}

Notes. ${ }^{(\star)}$ Of the thermal component.

of 3C 273 of $L_{\mathrm{j}} \approx 4 \times 10^{47} \mathrm{erg} \mathrm{s}^{-1}$ (Kataoka et al. 2002), $z_{\text {int }}$ found to be $\approx 3 \times 10^{17} \mathrm{~cm}$. The BLR luminosity of this source is $\approx 4 \times 10^{45} \mathrm{erg} \mathrm{s}^{-1}$ (Cao \& Jiang 1999), and its size $7 \times 10^{17} \mathrm{~cm}$ (Ghisellini et al. 2010), which implies that jet-cloud interactions can take place. The disc luminosity is high, $\approx 2 \times 10^{46} \mathrm{erg} \mathrm{s}^{-1}$, for typical photon energies $\approx 54 \mathrm{eV}$ (Grandi \& Palumbo 2004).

The non-thermal SED of the radiation generated by jet-cloud interactions in $3 \mathrm{C} 273$ is shown in Fig. 7. At $z_{\text {int }}$, the most important radiative processes are synchroton and SSC. The bolometric luminosities generated by these processes in one interaction at $z_{\text {int }}$ are $6 \times 10^{38} \mathrm{erg} \mathrm{s}^{-1}$ and $2 \times 10^{39} \mathrm{erg} \mathrm{s}^{-1}$, respectively. Given the strong radiation fields from the disc and the BLR, the emission above $\sim 10 \mathrm{GeV}$ is absorbed by photon-photon absorption, and the maximum of the emission occurs around $0.1-1 \mathrm{GeV}$. Given the estimated number of clouds in the BLR of 3C 273, $\sim 10^{8}$ (Dietrich et al. 1999), and the size of $R_{\mathrm{blr}} \approx 7 \times 10^{17} \mathrm{~cm}$, we find the filling factor to be $f \sim 3 \times 10^{-7}$. With this value of $f$, the number of clouds in the two jets results in $\sim 2 \times 10^{3}$ and $5 \times 10^{5}$ for both the minimum and maximum values (see Sect. 4). In the most optimistic case, the SSC luminosity would reach $2 \times 10^{44} \mathrm{erg} \mathrm{s}^{-1}$. This value is well below the observed luminosity by Fermi in the GeV range, $\sim 3 \times 10^{46} \mathrm{erg} \mathrm{s}^{-1}$ in the steady state and $\sim 1.7 \times 10^{47} \mathrm{erg} \mathrm{s}^{-1}$ in flares (Soldi et al. 2009). However, the detected emission is very likely of beamed origin and should mask any unbeamed radiation. However, powerful non-blazar AGNs (FR II galaxies) do not contain this beamed component, which makes it possible to detect $\mathrm{GeV}$ emission from jet-cloud interactions in these sources. In this case, given that many BLR clouds can interact with the jet simultaneously, the radiation should be steady.

\section{Summary and discussion}

We have studied the interaction of clouds with the base of jets in AGNs. Considering reasonable cloud and jet parameters, we have estimated the relevant dynamical timescales of these interactions, concluding that clouds can enter into the jet only above a certain height, $\sim z_{\text {int }}$. Below $z_{\text {int }}$, the jet is too compact and its $\mathrm{ram} /$ magnetic pressure will destroy the cloud before fully penetrating into the jet. When the cloud interacts significantly with the jet, strong shocks are generated and gamma rays can be produced with an efficiency that depends strongly on the bow-shock magnetic field.

For very high $B$-values (Poynting-flux dominated jets), the treatment performed here does not apply. In that case, $z_{\text {int }}$ could still be defined by adopting the jet magnetic pressure instead of the ram pressure. If a cloud entered into such a jet, particle acceleration in the bow shock could still occur due to, for instance, magnetic reconnection. A study of this case would require a completely different approach from the one presented here. In general, for bow-shock magnetic fields above equipartition with non-thermal particles, the IC channel and gamma ray production will be suppressed in favor of synchrotron emission, unless magnetic dissipation reduces the magnetic field sufficiently for IC to be dominant. Bow shock $B$-values well below equipartition with non-thermal particles produce significant gamma-ray emission. We note that the modeling of gamma rays from AGN jets uses to require relatively low magnetic fields (e.g. Ghisellini et al. 2010). Therefore, it may be that, even if the jet magnetic field were high at $z_{\text {int }}$, it could become small enough at greater heights thanks to bulk acceleration (e.g. Komissarov et al. 2007) or some other form of magnetic dissipation.

For very nearby sources, such as Cen A, the interaction of large clouds with jets may be detectable as a flaring event, although the number of these large clouds and thereby the duty cycle of the flares are difficult to estimate. Given the weak external photon fields in these sources, VHE photons can escape without experiencing significant absorption. Therefore, jet-cloud interactions in nearby FR I may be detectable in both the HE and the VHE range as flares with timescales of about one day. Studying this a radiation would provide information on the environmental conditions and the base of the jet in these sources.

In FR II sources, many BLR clouds may interact simultaneously with the jet. The number of clouds depends strongly on the cloud lifetime inside the jet, which could be of the order of several $t_{\mathrm{cs}}$. Nevertheless, we note that after cloud fragmentation many bow shocks may still form and efficiently accelerate particles if these fragments move more slowly than the jet. Since FR II sources are expected to exhibit high accretion rates, radiation above $1 \mathrm{GeV}$ produced in the jet base can be strongly attenuated by the dense disc and the BLR photon fields, although gamma rays below $1 \mathrm{GeV}$ should not be affected significantly. Since jet-cloud emission should be rather isotropic, it would be masked by jet beamed emission in blazar sources, although since powerful/nearby FR II jets do not display significant beaming, these objects may emit gamma rays from jetcloud interactions. In the context of AGN unification (Urry \& Padovani 1995), the number of non-blazar (radio-loud) AGNs should be much larger than that of blazars with the same $L_{\mathrm{j}}$. As shown in Fig. 5, close and powerful sources could be detectable by deep enough observations of Fermi. After few-year exposure times, a significant signal from these objects could be found, 
their detection providing strong evidence that jets are already strongly matter dominated at the bow shock regions, as well as physical information on the BLR and jet base region.

Acknowledgements. We thank the referee Elena Pian for constructive comments and sugestions. A.T.A. and V.B.-R. thanks Max Planck Institut fuer Kernphysik for kind hospitality and support. A.T.A. and G.E.R. are supported by CONICET and the Argentine agency ANPCyT (grant BID 1728/OC-AR PICT 2007-00848). V.B.-R. and G.E.R. acknowledge support by the Ministerio de Educación y Ciencia (Spain) under grant AYA 2007-68034-C03-01, FEDER fund

\section{References}

Abdo A. A., Ackermann, M., Ajello, M., et al. 2009, ApJ, 713, 1310

Abdo A. A., Ackermann, M., Ajello, M., et al. 2010, ApJ, 715, 429

Achterberg, A., Gallant, Y. A., Kirk, J. G., \& Guthmann, A. W. 2001, MNRAS, 328,393

Aharonian, F. A. 2002, MNRAS, 332, 215

Aharonian, F. A., Akhperjanian, A. G., Anton, G., et al. 2009, ApJ, 695, L40

Alexander, D. M., Hough, J. H., Young, S., et al. 1999, MNRAS, 303, L17

Araudo, A. T., Bosch-Ramon, V., \& Romero, G. E. 2009, A\&A, 503, 673

Araudo, A. T., Bosch-Ramon, V., \& Romero, G. E. 2010a, IJMPD, in press [arXiv: 0908.0926]

Araudo, A. T., Bosch-Ramon, V., \& Romero, G. E. 2010b, IJMPD, 19, 931

Bednarek, W., \& Protheroe R. J. 1997, MNRAS, 287, L9

Begelman, M. C., Blandford, R. D., \& Rees, M. J. 1984, Rev. Mod. Phys., 56, 255

Bell, A. R. 1978, MNRAS, 182, 147

Bentz, M. C., Peterson, B. M., Pogge, R. W., Vestergaard, M., \& Onken, C. A. 2006, ApJ, 644, 133

Bosch-Ramon, V. 2008, BAAA, 51, 293

Blake, G. M. 1972, MNRAS, 156, 67

Blandford, R. D., \& Königl A. 1979, ApJ, 232, 34

Boettcher, M. 2007, Ap\&SS, 309, 95

Cao, X., \& Jiang, D. R. 1999, MNRAS, 307, 802

Courvoisier, T. J.-L. 1998, A\&AR, 9, 1

Dar, A., \& Laor, A. 1997, ApJ, 478, L5

de Jager, O. C., Harding, A. K., Michelson, P. F., et al. 1996, ApJ, 457, 253
Dietrich, M., Wagner, S. J., Courvoisier, T. J.-L., Bock, H., \& North, P. 1999, A\&A, 351, 31

Evans, D. A., Kraft, R. P., Worrall, D. M., et al. 2004, ApJ, 612, 786

Ghisellini, G., Maraschi, L., \& Treves, A. 1985, A\&A, 146, 204

Ghisellini, G., Tavecchio, F., Foschini, L., et al. 2010, MNRAS, 402, 497

Grandi, P., \& Palumbo G. G. C. 2004, Sci, 306, 998

Hartman, R. C., Bertsch, D. L., Bloom, S. D., et al. 1999, ApJS, 123, 79

Israel, F. P. 1998, A\&AR, 8, 237

Jolley, E. J., Kuncic, Z., Bicknell, G. V., \& Wagner, S. 2009, MNRAS, 400, 1521

Junor, W., Biretta, J. A., \& Livio, M. 1999, Nature, 401, 891

Kaspi, S., Maoz, D., Netzer, H., et al. 2005, ApJ, 629, 61

Kaspi, S., Brandt, W. N., Maoz, D., et al. 2007, ApJ, 659, 997

Kataoka, J., Tanihata, C., Kawai, N., et al. 2002, MNRAS, 336, 932

Klein, R. I., McKee, C. F., \& Colella, P. 1994, ApJ, 420, 213

Komissarov, S. S., Barkov, M. V., Vlahakis, N., \& Königl, A. 2007, MNRAS, 380,51

Krolik, J. H., McKee, C. F., \& Tarter, C. B. 1981, ApJ, 249, 422

Liang, E. P. T., \& Thompson, K. A. 1979, MNRAS, 189, 421

Mannheim, K. 1993, A\&A, 269, 67

Marconi A., Schreider, E. J., Koekemoer, A., et al. 2000, ApJ, 528, 276

Mirabel, I. F., Laurent, O., Sanders, D. B., et al. 1999, A\&A, 341, 667

Mücke, A., \& Protheroe, R. J. 2001, Astrop. Phys., 15, 121

Paltani, S., \& Türler, M. 2005, A\&A, 435, 811

Penston, M. V. 1988, MNRAS, 233, 601

Peterson, B. M. 2006, LNP, 693, 77

Peterson, B. M., Bentz, M. C., Desroches, L.-B., et al. 2005, ApJ, 632, 799

Pian, E., Urry, C. M., Maraschi, L., et al. 1999, ApJ, 521, 112

Rees, M. J. 1987, MNRAS, 228, 47

Rieger, F. M., \& Aharonian, F. A. 2009, A\&A, 506, L41

Rieger, F. M., Bosch-Ramon, V., \& Duffy, P. 2007, Ap\&SS, 309, 119

Risaliti, G. 2010, IAU Symp., 267, 299

Risaliti, G., Elvis, M., \& Nicastro, F. 2002, ApJ, 571, 234

Romero, G. E., Combi, J. A., Perez Bergliaffa S. E., \& Anchordoqui, L. A. 1996, APh, 5, 279

Shakura, N. I., \& Sunyaev, R. A. 1973, A\&A, 24, 337

Shin, M.-S., Stone, J. M., \& Snyder, G. F. 2008, ApJ, 680, 336

Soldi, S., Beckmann, V., \& Türler, M. 2009, in 2009 Fermi Symp., eConf Proc. C091122, [arXiv:0912.2266v1]

Thomson, R. C. 1992, MNRAS, 257, 689

Tingay S. J., Jauncey, D. L., Reynolds, J. E., et al. 1998, AJ, 115, 960

Urry, C., \& Padovani, P. 1995, PASP, 107, 803

Wang, B., Inoue, H., Koyama K., \& Tanaka, Y. 1986, PASJ, 38, 685 